\title{
Role of Reconnection in AGN Jets
}

\author{
Maxim Lyutikov ${ }^{1,2,3}$ \\ ${ }^{1}$ McGill University, 3600 University street, Montreal, QC, Canada H3A 2T8 \\ ${ }^{2}$ MIT, 77 Massachusetts Avenue, Cambridge, MA 02139 \\ ${ }^{3}$ CITA National Fellow
}

\begin{abstract}
We discuss the possible role of reconnection in electro-magnetically dominated cores of relativistic AGN jets. We suggest that reconnection may proceed in a two-fold fashion: initial explosive collapse on the Alfvén time-scale of a current-carrying jet (which is of the order of the light crossing time) and subsequent slow quasi-steady reconnection. Sites of explosive collapse are associated with bright knots, while steady-state reconnection re-energizes particles in the "bridges" between the knots. Ohmic dissipation in reconnection layers leads to particle acceleration either by inductive electric fields or by stochastic particle acceleration in the ensuing electromagnetic turbulence.
\end{abstract}

Several arguments point to the possibility that in the inner cores of AGN jets the energy is transported mostly by Poynting flux (see, e.g., Blandford 2002). Perhaps the two strongest arguments in favor of magnetically dominated jets are the extremely strong collimation of some jets (e.g., Pictor A) observed on a scale of tens of kiloparsecs and a very short time-scale variability observed at $\mathrm{TeV}$ energies in blazars. Unlike magnetically dominated jets, the hydrodynamic jets were expected to be strongly influenced by interaction with the surrounding which should lead to generation of oblique shocks, strong dissipation in the jets and a loss of collimation. Apparently this does not happen, at least in some jets. An alternative possibility is that jets consist of a strongly magnetically dominated relativistic core, weakly relativistic sheath and later, as the interaction with the external medium becomes important, a cocoon of the entrained material.

Recent observations of $\mathrm{TeV}$ emission from blazars with a very short time scale variability stressed once again the necessity for in situ acceleration - the cyclotron decay times for the X-ray and especially $\gamma$-ray emitting electrons are an order of magnitude smaller than the light time travel from the core (and often smaller than the light crossing time of the emitting region itself). The two popular models of jet composition - leptonic and ionic - both have 
difficulties in explaining the fast variability (a problem for ion-dominated jets, since cyclotron times are very long for ions) and total energy content (since leptons suffer strong radiative losses at the source due to radiation drag).

Magnetically dominated jets are free from both of these constraints. Magnetic energy is both "quiet" (is not subject to the compactness problem and thus does not suffer from radiative losses at the source) and of "high quality" - the energy stored in a low entropy electro-magnetic outflows can be effectively converted into high frequency electro-magnetic radiation far away from the source. Thus, the assumption of magnetically dominated jets implies that the energy of the central source is released in magnetic form (e.g., by the Blandford-Znajek mechanism), transported as a Poynting flux to large distances and then dissipated by current instabilities.

Other properties of magnetic fields may argue in favor of magnetically dominated jets. (1) Magnetic fields are strongly non-linear systems: slow evolution during which magnetic stresses build-up may lead to accumulation of a large amount of free energy which is released on a short time scale (of the order of the Alfvén time scale) as the system crosses stability threshold. (2) Magnetic field dissipation is usually strongly intermittent (as is exemplified by solar flares) and may be repetitive in time and/or in space. (3) Magnetic field dissipation may be internal and does not necessarily lead to the global disruption of a system. (4) Magnetic field dissipation leads to particle acceleration producing power-law distribution of accelerated particles.

On the other hand, there are definite problems with reconnection models. Reconnection physics is highly uncertain: it depends crucially on the kinetic and geometric properties of the plasma, which is very hard to test observationally (e.g., Litvinenko 1999). Most importantly, various instabilities (based on inertial or cyclotron effects, on ions or electrons) often seem to account equally well (with astrophysical accuracy) for the observed phenomena. This is an important uncertainty since the principle scale of the reconnection region (related, for example, to electron or ion skin depth, Larmor radius or magnetic Debye radius) is crucial in determining the rate of reconnection (e.g., Birk \& Lesch 2000). Thus, it is hard to produce a testable result that would give a decisive answer to the question whether reconnection is important or not in the AGN jets. This situation may be contrasted with the shock acceleration schemes, where a qualitatively correct result for the spectrum of accelerated particles can be obtained from simple macroscopic considerations.

Currently, the most popular models of AGN jets assume that the dissipation is due to internal shocks related to time-depend source activity. How different are the observational effects of reconnection from those of the internal shocks models? Two separate questions are important here: that of particle acceleration (spectra of particles, cut-off energies, etc) and emission genera- 
tion (synchrotron, IC, SSC, etc). Emission generation is likely to be the same in reconnection models, so that most of the well detailed radiation models will still hold (modulo, perhaps, the assumption of the equipartition magnetic field). On the other hand, the acceleration mechanism is very different, but hardly distinguishable observationally - both internal shocks and reconnection regions represent transient internal dissipative regions, which heat the plasma and accelerate particles. Spectra of accelerated particles are powerlaws in both cases (power-law distributions are naturally produced in shock acceleration; in the case of reconnection there is no theoretical expectation of power-laws, but they are observed both on the Sun and in numerical experiments). A possible distinction between shock and reconnection acceleration is the value of the power-law spectral index. Larrabee et al. (2002) have shown that in relativistic reconnection the spectrum of accelerated particles may be very hard, $d n / d \gamma \sim \gamma^{-1}$; this is prohibitively hard for shock acceleration. In fact, such hard power-laws may be needed to explain the $\mathrm{TeV}$ emission from blazars and its propagation through the IR background.

We expect that in a relativistically moving jet the reconnection may begin at distances $r \geq R_{S} \Gamma^{2}$ ( $R_{S}$ is the Schwarzschild radius of the central black hole $(\mathrm{BH})$ ), which for a $\mathrm{BH}$ of $10^{8} M_{\odot}$ and $\Gamma \sim 30$ gives $R \geq 10^{16} \mathrm{~cm}$. This is a fairly large distance, so the compactness of the emitting region is small and the high energy photons are not likely to be scattered inside the emitting region.

Reconnection is expected to develop in several stage. (1) Slow (quiet) MHD evolution, build-up of magnetic stresses, $r \leq R_{S} \Gamma^{2} \sim 10^{16} \mathrm{~cm}$. (2) Explosive MHD collapse (sometimes called magnetic detonation or MHD catastrophe) developing on an Alfvén time-scale and leading to formation of current sheets. (3) Steady state reconnection.

Slow evolution. Jets are launched in the vicinity of a BH-disk system. In the process of acceleration they become super-fastmagnetosonic and thus causally disconnected from the source. As the relativistic flow expands it cannot adjust its lateral balance on the expansion time scale due to effective freezing of lateral dynamics in relativistically moving jets. This leads to the build-up of uncompensated stresses in the flow - a jet is in a strongly non-equilibrium state.

Explosive collapse. At distances $r \geq R_{S} \Gamma^{2}$ a jet starts to evolve laterally; large uncompensated hoop stresses directed towards the axis result in a jet compression. We propose that at this point the Poynting flux-dominated core of a jet undergoes an electro-magnetic explosive collapse, similar to explosive growth in Z-pinches, high- $\beta$ TOKAMAKs disruptions, geomagnetic substorms and solar flares. Alternatively, enhanced dissipation may be due to the onset of current instabilities that generate plasma turbulence and result in anomalous 
resistivity.

Steady state reconnection. Explosive collapse drives a reconnection process. The steady-state rates of reconnection in AGN jets maybe estimated using the formulation of relativistic reconnection by Lyutikov \& Uzdensky (2002), who found that in a strongly magnetized plasma the inflow velocity is $\beta_{\text {in }} \sim \sqrt{\sigma / S}$ (for $\sigma \leq S$ ), where $\sigma \gg 1$ is the ratio of magnetic to plasma energy densities and $S$ is the Lundquist number $S=L c / \eta \gg 1, L$ is the size of the reconnection layer and $\eta$ is the resistivity. The outflowing plasma is always relativistic, $\gamma_{\text {out }} \sim \sigma \gg 1$. Assuming that the dominant dissipation process is related to the ion Larmor radius $r_{L}$ and using Bohm's prescription for resistivity, $\eta \sim c r_{L}$ we find that typically the reconnection inflow velocities are non-relativistic, $\beta_{\text {in }} \sim 0.01\left(\sigma / 10^{4}\right)^{1 / 2}$.

Particle acceleration in reconnection regions may occur in a variety of ways, through DC acceleration, Fermi-type acceleration in MHD or electro-magnetic turbulence and shock acceleration at the different kinds of MHD shocks produced near the reconnection region. Simple qualitative estimates may be done assuming that particle acceleration is due to DC electric fields and is balanced by synchrotron radiative losses. One then finds the maximum Lorentz factor and the maximum energies for synchrotron and inverse Compton emission

$$
\gamma \sim \sqrt{\beta_{\text {in }}} \sqrt{\frac{c}{r_{e} \omega_{B}}}, \quad \epsilon_{\text {synch }} \sim \beta_{\text {in }} \frac{\hbar c}{r_{e}} \sim 20 \beta_{\text {in }} \mathrm{MeV}, \quad \epsilon_{\mathrm{IC}} \sim \gamma m c^{2} \sim 100 \mathrm{TeV}(1)
$$

where $r_{e}=e^{2} / m c^{2}$ and $\omega_{B}=e B / m c$. These values are able to accommodate most observational constraints on the AGN emission properties.

Given all the possible advantages that reconnection may bring, can one "prove" that it plays an important role in AGN jets? Probably not from first principles. We expect that reconnection will become an accepted model for jet re-energization either by some particular analogy to solar phenomena (e.g., coherent radio emission correlated with $\mathrm{X}$-ray and $\gamma$-rays) or by exclusion (e.g., spectra prohibitively hard for shock acceleration). Some of the observations may already be in place: some knots have very hard spectra (e.g., the knot A1 of 3C 273 has $\alpha \sim 0.6$, which requires an electron acceleration spectrum with $p=1.2$, a challenge to any shock acceleration model).

\section{References}

[1] Birk, G. T., Lesch, H., 2000, ApJ Lett., 530, 77

[2] Blandford, R. D., 2002, in Lighthouses of the Universe, Gilfanov M, Sunyaev, R, Churazov, E, eds., p. 381

[3] Larrabee, D.A., Lovelace R.V.E., Romanova M.M., astro-ph/0210045

[4] Litvinenko, Y. E., 1999, A\&A, 349, 685

[5] Lyutikov, M., Uzdensky D., 2002, astro-ph/0210206

[6] Priest, E., Forbes, T., 2000, "Magnetic reconnection : MHD theory, applications",

Cambridge University Press 